\title{
Possible Role of Regulatory B Cells in Different Behçet's Disease Phenotypes and Therapies: First Report from Egypt
}

This article was published in the following Dove Press journal: Journal of Inflammation Research

\begin{abstract}
Helal F Hetta, (D) ${ }^{1,2} *$
Alaa AA Mohamed, (D) ${ }^{3, *}$

Asmaa M Zahran, ${ }^{4, *}$

Safaa A Mahran, ${ }^{3}$

Marwa MY Sayed, ${ }^{5}$

Mohamed GA Saleh, ${ }^{6}$

Khaled Abdelazeem, ${ }^{6}$ Gaber

El-Saber Batiha, ${ }^{7}$ Salim Al-Rejaie,

Yasir Waheed, ${ }^{9}$

Khalid Muhammad, ${ }^{10}$

Manal M Hassanien ${ }^{3}$

'Department of Medical Microbiology and Immunology, Faculty of Medicine, Assiut University, Assiut, Egypt; ${ }^{2}$ Department of Internal Medicine, University of Cincinnati College of Medicine, Cincinnati, OH, USA; ${ }^{3}$ Rheumatology and Rehabilitation Department, Faculty of Medicine, Assiut University, Assiut, Egypt; ${ }^{4}$ Clinical Pathology Department, South Egypt Cancer Institute, Assiut University, Assiut, Egypt; ${ }^{5}$ Department of Cardiology, Faculty of Medicine, Assiut University, Assiut, Egypt; ${ }^{6}$ Department of Ophthalmology, Faculty of Medicine, Assiut University, Assiut, 71515, Egypt; ${ }^{7}$ Department of Pharmacology and Therapeutics, Faculty of Veterinary Medicines, Damanhour University, Damanhur, 225। I, Egypt; ${ }^{8}$ Department of Pharmacology \& Toxicology, College of Pharmacy, King Saud University, Riyadh, Saudi Arabia; ${ }^{9}$ Foundation University Medical College, Foundation University Islamabad, Islamabad, 44000, Pakistan; ${ }^{10}$ Department of Biology, College of Science, United Arab Emirates University, Al Ain, I555I, United Arab Emirates

*These authors contributed equally to this work
\end{abstract}

Correspondence: Helal F Hetta Department of Medical Microbiology and Immunology, Faculty of Medicine, Assiut University, Assiut, 71515, Egypt

Email helalhetta@aun.edu.eg
Background and Aim: The data about the role of regulatory B cells (Breg) in Behcet Disease $(\mathrm{BD})$ are scarce. We aimed to evaluate the frequency of total B lymphocytes and Breg cells in different BD phenotypes and therapies attempting to unravel their function.

Methods: This cross-sectional study included 35 BD patients and 39 healthy controls (HCs). The demographic data of the study subjects were collected including age and gender. Current medications including disease-modifying anti-rheumatic drugs (DMARDs) were recorded. All patients underwent testing for baseline laboratory investigations including full blood count, liver and kidney function tests, erythrocyte sedimentation rate (ESR) by Westergren blot and C-reactive protein (CRP). Measurement of the total B lymphocytes and their subtypes B regulatory lymphocytes by flow cytometric assay. Assessment of BD activity was done using the revised Behçet's Disease Current Activity Form (BDCAF) 2006 and Behçet's Syndrome Activity Score (BSAS) ${ }^{1111111111}$. All participants were assessed for the presence of erectile dysfunction using the International Index of Erectile Function (IIEF-5 score), and for depression using the Beck Depression Inventory.

Results: A dramatic drop in the number of B cells, total and regulatory, was observed in the patients compared to the HCs. Regulatory cells (Bregs) tend to be upregulated with genital ulcers or vascular disease. Bregs but not B lymphocytes were associated with BSAS and ESR. Neither the total B lymphocytes nor the Bregs correlated with CRP or the sexual function or depression scores. Of all the used medications, low-dose aspirin was seen with markedly high Bregs proportions.

Conclusion: This study supports the role of B cells in BD pathogenesis and strongly suggests a possible role for Bregs in the resolution of different BD manifestations.

Keywords: Behcet's disease, B lymphocytes, Breg

\section{Introduction}

Behçet's disease (BD) is a chronic multi-organ vasculitis characterized by recurrent oral and genital aphthae, skin lesions, widespread thrombosis and aneurysmal formation, and serious involvement of the eyes and central nervous system. ${ }^{2}$ The evolution of the disease is incompletely understood, and different mechanisms were hypothesised to take place in the development of the disease, particularly, the environmental triggers. ${ }^{3}$ Bacterial infections and heat shock proteins have been thought to play an important role in the initiation of the disease via molecular mimicry. ${ }^{4,5}$ Furthermore, the genetic factor of the disease was strongly linked to the presence of HLA-B51. ${ }^{6}$ However, an array of single-nucleotide polymorphisms 
encoding for cytokines, chemokines, receptors and signal transducers were also detected in BD patients, and were linked to increased susceptibility to the disease. ${ }^{7}$

The innate and adaptive immune cells integrate into the pathogenesis of BD. It appears that the inflammatory process is initiated by neutrophils and perpetuated by y $\partial$ $\mathrm{T}$ cells which induce strong $\mathrm{T}$ helper (Th)-1, Th-2 and Th17 responses. ${ }^{8}$ Interestingly, the gene expression analysis of BD patients and healthy controls revealed the modulation of a range of genes involved in biological processes in BD, such as inflammation, apoptosis, angiogenesis, blood coagulation, vascular damage, signalling pathways, and immune responses, particularly, in innate immune cells, Th17 cells and B cells. ${ }^{9}$

Earlier, evidence of B cell involvement in the disease had grown through the observation of increased specific B cell subsets in BD compared to healthy controls and other autoimmune diseases, ${ }^{10}$ and the detection of abnormalities in $\mathrm{B}$ cell functions which were demonstrated to be associated with disease pathogenesis and disease activity. ${ }^{11}$ Moreover, $\mathrm{B}$ cells were detected in active mucosal ulcers, ${ }^{12}$ and the depletion of B cells was shown to be effective in improving the ocular disease. ${ }^{13}$ The regulatory function of Breg in autoimmune diseases was extensively studied in animal models. It was found to act as antigenpresenting cells and produce autoantibodies leading to targeted tissue damage. Thus, it was claimed to play a pathogenic role in different autoimmune diseases as rheumatoid arthritis, lupus, autoimmune diabetes mellitus and autoimmune encephalomyelitis. ${ }^{14-20}$

Yet, the regulatory role of $\mathrm{B}$ cells in BD has not been thoroughly investigated, ${ }^{21}$ and their role in different disease presentations has not been studied. Therefore, the aim of the current study is to identify the proportions of total B lymphocytes and their regulatory subset in different BD phenotypes and therapies attempting to unravel their function in BD.

\section{Methods}

\section{Subjects and Methods}

This observational, cross-sectional study was conducted on patients attending the Rheumatology Clinics in Assiut University Hospitals, Egypt. It was approved by the ethical committee of Assiut Faculty of Medicine in accordance with the declaration of Helsinki. Clinical trial identification number NCT04376411. Written consents were collected from all the participants before recruitment. We enrolled 35 patients aged $\geq 18$ years who fulfilled the diagnosis of $\mathrm{BD}$ after scoring four or more points of the International Criteria for Behçet's Disease (ICBD). ${ }^{22}$ Thirty-nine age and sex-matched healthy controls (HCs) were also recruited after being consented. The patients and healthy controls were informed about the purpose of the trial.

\section{Clinical and Laboratory Assessment}

After full history taking and clinical examination, assessment of the patients' disease activity was done using the revised Behçet's Disease Current Activity Form (BDCAF) 2006 of the original BDCAF ${ }^{23,24}$ And Behçet's Syndrome Activity Score (BSAS). ${ }^{1}$

Current medications including disease-modifying antirheumatic drugs (DMARDs) were recorded. All patients underwent testing for baseline laboratory investigations including full blood count, liver and kidney function tests, erythrocyte sedimentation rate (ESR) by Westergren blot and C-reactive protein (CRP). An expert cardiologist performed a full cardiac examination to exclude cardiac involvement including blood pressure and left ventricular function. Patients and HCs were assessed for the presence of erectile dysfunction using the International Index of Erectile Function (IIEF-5 score), ${ }^{25}$ and for the presence of depression using the Beck Depression Inventory. ${ }^{26}$

\section{Flow-Cytometric Assay}

Using flow cytometry, circulating Bregs were detected using PE-conjugated CD38, FITC-conjugated CD24 (Bioscience, USA) and PerCP-conjugated CD19 (BD Bioscience, USA).

One hundred microliters of the blood sample was incubated with $10 \mu \mathrm{L}$ of CD24, CD38 and CD19 for 20 minutes at $4 \mathrm{C}$ in the dark. Following incubation, red blood cells lysis, washing and analysis by FACS Calibur flow cytometry with CellQuest software (Becton Dickinson Biosciences, USA) were done. An isotypematched negative control was used for each sample. Forward and side scatter histogram was used to define the lymphocytes population. Then, $\mathrm{CD} 19^{+} \mathrm{B}$ cells were gated. Then, the expression of CD38 and CD24 on the $\mathrm{CD} 19^{+} \mathrm{B}$ cells was detected. Bregs were identified as $\mathrm{CD} 19^{+} \mathrm{CD} 24^{+ \text {hi }} \mathrm{CD} 38^{+ \text {hi }}$ cells (Figure 1).

\section{Statistical Analysis}

The data were subjected to normality tests and were presented as means and standard deviations or medians and 
A

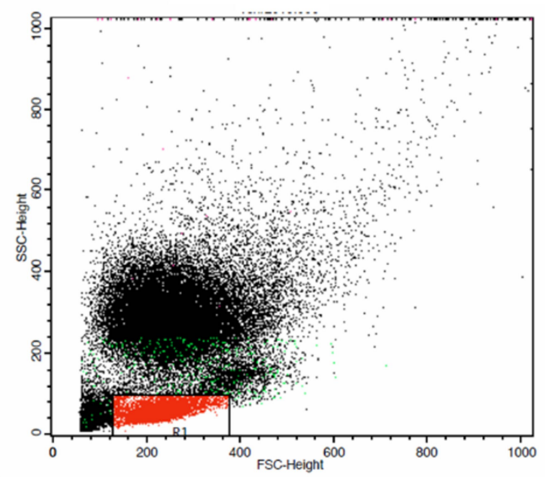

B

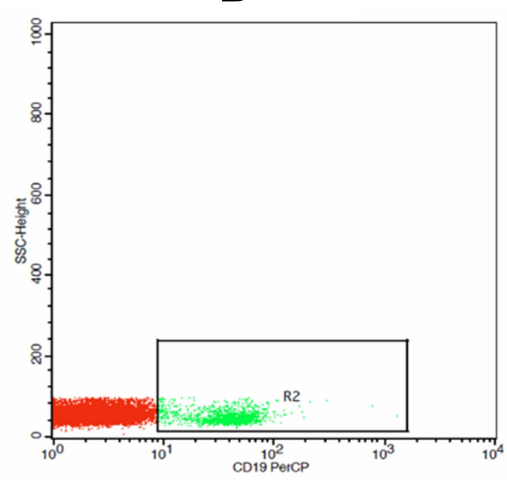

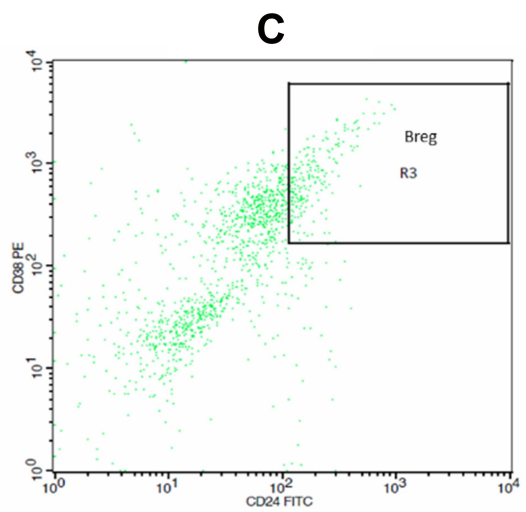

Figure I Flow cytometric detection of regulatory B cells.

Notes: (A) Forward and side scatter histogram was used to define the lymphocytes population (RI). (B) The CDI9+ cells (R2) were assessed within the lymphocyte population. (C) The expression of CD24 and CD38 was assessed in CD19+ cells to define CDI9+CD24 $4^{\text {thigh }}$ CD $38^{\text {thigh }}$ cells (regulatory $B$ cells).

interquartile ranges as appropriated. For categorical variables, data were presented as numbers and percentages. To compare between two or more groups, Independent $T$ test, Mann Whitney $U$-test and Kruskal-Wallis test were used as appropriate. Chi-square test and Fisher's exact test were employed to compare categorical data as appropriate. Spearman's rho correlation coefficient was used to determine the correlations between groups. P-values less than 0.05 were considered significant. The statistical program used for all statistical analyses is the Statistical Package for Social Science (SPSS) version 24 (SPSS Inc.; Chicago, IL, USA).

\section{Results}

A total number of 35 BD patients and 39 health controls (HCs) were gathered. The mean age of the participants' was $35.2 \pm 7.6$ for patients and $36.7 \pm 5.7$ for HCs. Twentysix of the patients and 27 of the HCs were of the male gender. Comparable numbers of the patients and HCs were smokers (22.9\% and $30.8 \%$, respectively). The clinical and immunological profile of the study subjects, including the proportions of total and regulatory B cells, and therapy of the BD patients were shown in Tables 1 and 2. A dramatic drop in the number of B cells, total and regulatory, was observed in the patients compared to the HCs. A nonsignificant correlation was found between the frequency of both B lymphocytes and B regs with all of the following: heart rate, systolic blood pressure and diastolic blood pressure $(\mathrm{P}>0.05)$.

Studying the trends of total and regulatory B cells in different disease phenotypes revealed a tendency of the $\mathrm{B}$ regulatory cells (Bregs) to be upregulated in different involvements, in particular, in the presence of genital ulcers or vascular disease despite the markedly low number of B cells in the arterial diseases as demonstrated in Table 3. On the contrary, there were no significant differences in either total B lymphocytes or Bregs in the presence or absence of ocular involvement or in different ocular manifestations. Furthermore, they showed no significant differences with favourable or unfavourable responses, to first-line therapy, or with the number of relapses per year.

Though both BSAS and ESR did not correlate with each other (data not shown), there were significant relationships between the proportions of total B cells, on one side, and BSAS ( $\mathrm{rho}=-0.36, \mathrm{p}=0.033$ ) and ESR $(\mathrm{rho}=0.34$, $\mathrm{p}=0.047$ ), on the other side, as illustrated in Figure 2. On the other hand, BSAS and ESR displayed no associations with Bregs. Noticeably, neither the total B lymphocytes nor the regulatory subset correlated with CRP or the sexual function or depression scores.

Studying the changes in B cells linked to the therapeutic profile of the patients revealed no substantial changes in B lymphocytes or Bregs with the administration of different DMARDs. Nevertheless, the use of low-dose aspirin was seen with markedly high Bregs proportions, and there was a similar trend with the use of anticoagulants despite the lack of a significant difference as illustrated in Figure 3.

As all patients were on different doses of prednisolone, $\mathrm{B}$ cell numbers were tested against the dose and duration of corticosteroid administration. It was observed that the doses of prednisolone correlated positively with the proportions of Bregs ( $r h o=0.394, p=0.019)$ but not with those of total B lymphocytes ( $\mathrm{rho}=0.081, \mathrm{p}=0.65$ ). The durations 
Table I Clinical and Immunological Characteristics of the Study Subjects

\begin{tabular}{|c|c|c|c|}
\hline & $\begin{array}{c}\text { BD Patients } \mathbf{N}=35 \\
N(\%)\end{array}$ & Control $\mathbf{N}=39$ & p-value \\
\hline Arterial disease & $17(48.6 \%)$ & NA & NA \\
\hline DVT & $16(45.7 \%)$ & NA & NA \\
\hline GIT & $10(28.6 \%)$ & NA & NA \\
\hline Skin lesions/Pathergy test positive/Oral ulcers & $35(100 \%)$ & NA & NA \\
\hline Genital ulcers & $16(45.7 \%)$ & NA & NA \\
\hline Urethritis, n (\%) & $6(17.1 \%)$ & NA & NA \\
\hline Ocular disease & & NA & NA \\
\hline - Anterior uveitis & $2(5.7 \%)$ & & \\
\hline - Pan uveitis and retinal vasculitis & $6(17.1 \%)$ & & \\
\hline - Pan uveitis and retinal vasculitis with optic neuritis/atrophy & $12(34.3 \%)$ & & \\
\hline - No ocular signs & $15(42.9 \%)$ & & \\
\hline Arthralgia/arthritis & $20(57.1 \%)$ & NA & NA \\
\hline Headache & $5(14.3 \%)$ & NA & NA \\
\hline Seizures & 0 & NA & NA \\
\hline Cognitive dysfunction & $6(17.1 \%)$ & NA & NA \\
\hline Peripheral neuropathy & $10(28.6 \%)$ & NA & NA \\
\hline Psychosis & 0 & NA & NA \\
\hline Inflammatory back pain & 14 (40\%) & NA & NA \\
\hline Activity within the past four weeks (BDCAF 2006) & $21(60 \%)$ & NA & NA \\
\hline Erectile dysfunction (IIEF-5 score) \# & $15(9-20)$ & $20(20-25)$ & $<0.001 *$ \\
\hline Depression score (Beck questionnaire) \# & $13(8-19)$ & $10(7-14)$ & $0.042 *$ \\
\hline BSAS \# & $30(30-60)$ & NA & NA \\
\hline ESR\# & $25(17-34)$ & $20(16-26)$ & $0.02 *$ \\
\hline CRP§ & $14.4 \pm 9.8$ & $2 \pm 3.4$ & $<0.001 *$ \\
\hline B lymphocytes \% § & $11.1 \pm 1.9$ & $14.2 \pm 3$ & $<0.001 *$ \\
\hline B reg.\% $\S$ & $1.9 \pm 0.5$ & $3.3 \pm 1.4$ & $<0.001 *$ \\
\hline
\end{tabular}

Notes: Independent $T$ test, Mann-Whitney U-test and Chi-square test were used as appropriated. *Significance was considered when P $\leq 0.05$

Abbreviations: BD, Behcet's disease; SD, standard deviation; DVT, deep venous thrombosis; GIT, gastrointestinal manifestations; BDCAF, Behçet's Disease Current Activity Form; \#, median (interquartile range); §, mean \pm standard deviation; BSAS, Behçet's Syndrome Activity Score; ESR, erythrocyte sedimentation rate; CRP, C-reactive protein.

Table 2 DMARDs Administration in BD Patients

\begin{tabular}{|l|c|}
\hline Drug Used & $\begin{array}{c}\text { BD Patients N=35, } \\
\mathbf{N}(\%)\end{array}$ \\
\hline Prednisolone doses & \\
$10 \mathrm{mg}$ & $9(25.7 \%)$ \\
$20 \mathrm{mg}$ & $1 \mathrm{I}(31.4 \%)$ \\
$30 \mathrm{mg}$ & $7(20 \%)$ \\
$40 \mathrm{mg}$ & $7(20 \%)$ \\
$60 \mathrm{mg}$ & $1(2.9 \%)$ \\
Prednisolone duration (years) \# & $4(3-5)$ \\
\hline CYC & $8(22.9 \%)$ \\
CS & $11(31.4 \%)$ \\
MTX & $9(25.7 \%)$ \\
AZA & $13(37.1 \%)$ \\
LDA & $12(34.3 \%)$ \\
Anticoagulant & $4(11.4 \%)$ \\
\hline
\end{tabular}

Abbreviations: DMARDS, disease-modifying anti-rheumatic drugs; BD, Behçet's disease; \#, median (interquartile range); CYC, cyclophosphamide; CS, cyclosporine; MTX, methotrexate; AZA, azathioprine; LDA, low dose aspirin. of corticosteroid administration did not correlate with either total B lymphocytes or Bregs. Unfortunately, within the recruited patients, none of them had started biologic therapy prior to enrolment; therefore, the effect of its commencement was not studied.

\section{Discussion}

Multiple innate and adaptive immunological responses are proven to be implicated in the pathogenesis of BD. ${ }^{9}$ However, the participation of these different responses to initiate diverse manifestations is still unclear particularly the role of $B$ cells. The current study aims to identify the aberrations in B lymphocytes and B regs in different BD manifestations and therapy.

In this study, patients diagnosed with BD had significantly lower proportions of $B$ cells and a remarkably lower $\mathrm{B}$ regulatory fraction in the peripheral blood compared to 
Table 3 The Differences in Total B Lymphocytes and B Regulatory Subset with Different Disease Characteristics

\begin{tabular}{|c|c|c|c|c|c|c|}
\hline & $\begin{array}{l}\% \text { of B Cells } \\
\text { in (+) Group } \\
\text { Median (IQR) }\end{array}$ & $\begin{array}{l}\% \text { of B Cells } \\
\text { in (-) Group } \\
\text { Median (IQR) }\end{array}$ & p-value & $\begin{array}{l}\text { \% of B Reg. in } \\
\text { (+) Group } \\
\text { Median (IQR) }\end{array}$ & $\begin{array}{l}\% \text { of B Reg. in } \\
\text { (-) Group } \\
\text { Median (IQR) }\end{array}$ & p-value \\
\hline Activity within 4 weeks & $12(1 \mathrm{I}-12.4)$ & $11.3(10.4-12)$ & 0.14 & $1.86(1.5-2.3)$ & $1.8(1.5-2.2)$ & 0.5 \\
\hline Arterial disease & $11(9-12)$ & $12(11.3-12.5)$ & $0.013^{*}$ & $2.2(1.7-2.4)$ & $1.7(1.5-2)$ & 0.067 \\
\hline DVT & II.8 (I0.7-I2.I) & $11.5(10.4-12.4)$ & 0.8 & $2.1(1.8-2.4)$ & $1.6(1.4-2.1)$ & 0.052 \\
\hline GIT & $12(11.3-12.4)$ & $11.4(10.4-12.2)$ & 0.3 & $1.9(1.7-2.6)$ & $1.8(1.5-2.2)$ & 0.4 \\
\hline Genital ulcers & $11.6(10.2-12.1)$ & $11.9(10.8-12.4)$ & 0.4 & $2.1(1.7-2.5)$ & $1.7(1.4-1.9)$ & $0.04 *$ \\
\hline Urethritis & $11.8(11.2-12.2)$ & $11.6(10.4-12.2)$ & 0.7 & $2.5(2.2-2.6)$ & $1.8(1.5-2)$ & $0.002 *$ \\
\hline Arthralgia/arthritis & $11.9(10.5-12.6)$ & $11.4(10.4-12.1)$ & 0.46 & I.8 (I.4-2.4) & $1.86(1.6-2.1)$ & 0.88 \\
\hline Headache & $12.2(\mid 1.2-12.4)$ & $11.6(10.4-12.2)$ & 0.57 & $2(1.6-2.16)$ & $1.8(1.5-2.2)$ & 0.84 \\
\hline PN & $11.3(7.2-12)$ & $12(10.6-12.4)$ & 0.12 & $1.9(1.5-2.3)$ & $1.86(1.5-2.2)$ & 0.76 \\
\hline Cognitive dysfunction & $12.2(\mid 1.2-12.4)$ & $11.5(10.4-12)$ & 0.38 & $2(1.6-2.16)$ & $1.8(1.5-2.2)$ & 0.69 \\
\hline Inflammatory back pain & $11.6(10.6-12.2)$ & $11.9(10.4-12.2)$ & 0.93 & $1.8(1.5-2.2)$ & $1.86(1.6-2.2)$ & 0.82 \\
\hline Smoking & $10.6(9.4-12.2)$ & $11.9(10.8-12.2)$ & 0.32 & $2.3(1.5-2.5)$ & $1.8(1.5-2.1)$ & 0.24 \\
\hline Family history of AIDs & $12(10.4-12.3)$ & $11.5(10.4-12.2)$ & 0.92 & $2(1.7-2.4)$ & $1.8(1.4-2.2)$ & 0.3 \\
\hline
\end{tabular}

Note: Mann-Whitney U-test was used to identify the significance between the groups. *Significance was considered when $\mathrm{P} \leq 0.05$.

Abbreviations: IQR, interquartile range; DVT, deep venous thrombosis; GIT, gastrointestinal manifestations; PN, peripheral neuropathy; AID, autoimmune diseases.

the healthy controls. Further studying of changes in their numbers in different disease manifestations revealed a marked drop in B lymphocytes in arterial diseases. However, there was a trend of $\mathrm{B}$ regs to be upregulated in the presence of major vascular diseases, genital ulcers and urethritis.

Consistently, it was noticed that B regs were higher in patients who were commenced on LDA and possibly in those who received anticoagulants, as those medications were used exclusively as adjuvant therapy in major vascular diseases. On the other hand, the use of

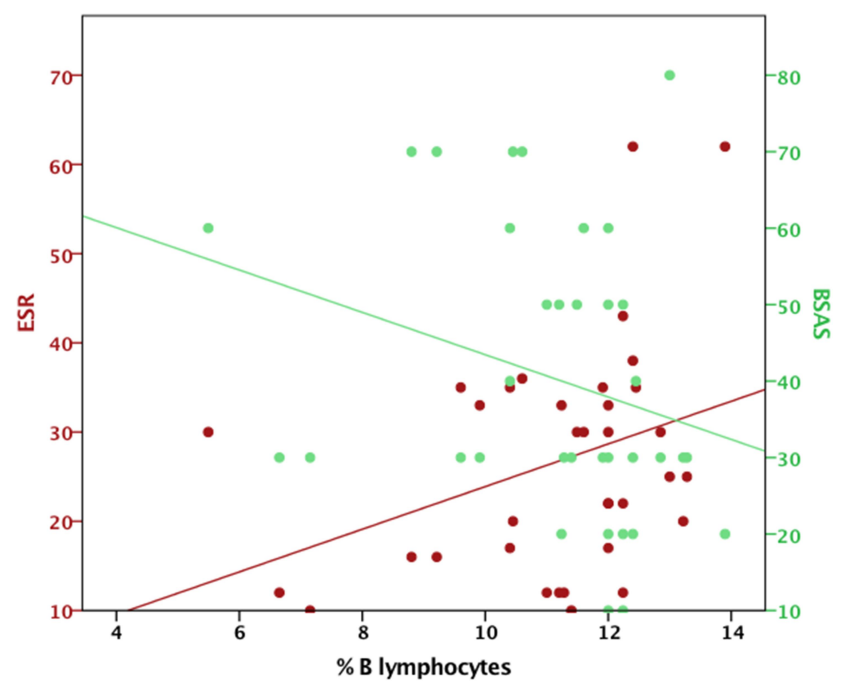

Figure 2 The relationship of B lymphocytes with ESR and BSAS scores. Abbreviations: ESR, erythrocyte sedimentation rate; BSAS, Behçet's Syndrome Activity Score. immunosuppressives, which were indicated for different disease manifestations, did not exhibit significant variations in B regs percentages. Actually, all the used immunosuppressing medications were working on the $\mathrm{T}$ cells and none of the patients in this study had received medications that target the B cells like Rituximab.

Noticeably, the total proportions of B cells correlated negatively with the scores of disease activity, whereas they correlated positively with ESR. Moreover, the increment in the dose of prednisolone was associated with an increment in B regs numbers.

In line with our findings, a reduction in numbers of $\mathrm{B}$ cells in $\mathrm{BD}$ patients compared to HCs has been reported before $^{21}$ and attributed to a drop in certain subsets such as $\mathrm{CD} 27^{+}$memory B cells. ${ }^{12}$ Additionally, a decline in the peripheral blood $\mathrm{B}$ regs of the patients compared to the controls was also observed by Yoon et al despite the lack of a significant difference in their study. ${ }^{21} \mathrm{~B}$ regs play an immunomodulatory and suppressive role against autoinflammation and auto-reactivation, as was noticed in other diseases, via IL-10 dependent and independent mechanisms, through maintaining $\mathrm{T}$ regulatory cells and negatively regulating $\mathrm{T}$ cell differentiation. ${ }^{17,18,27}$ Thus, the observed drop in B regs numbers may be accounting for the increased risk of acquiring BD. An inverse relationship between the total number of B cells and the disease activity scores was present in the current study which was also reported by van der Houwen et al who observed shifting of $B$ cells from the blood to the sites of 


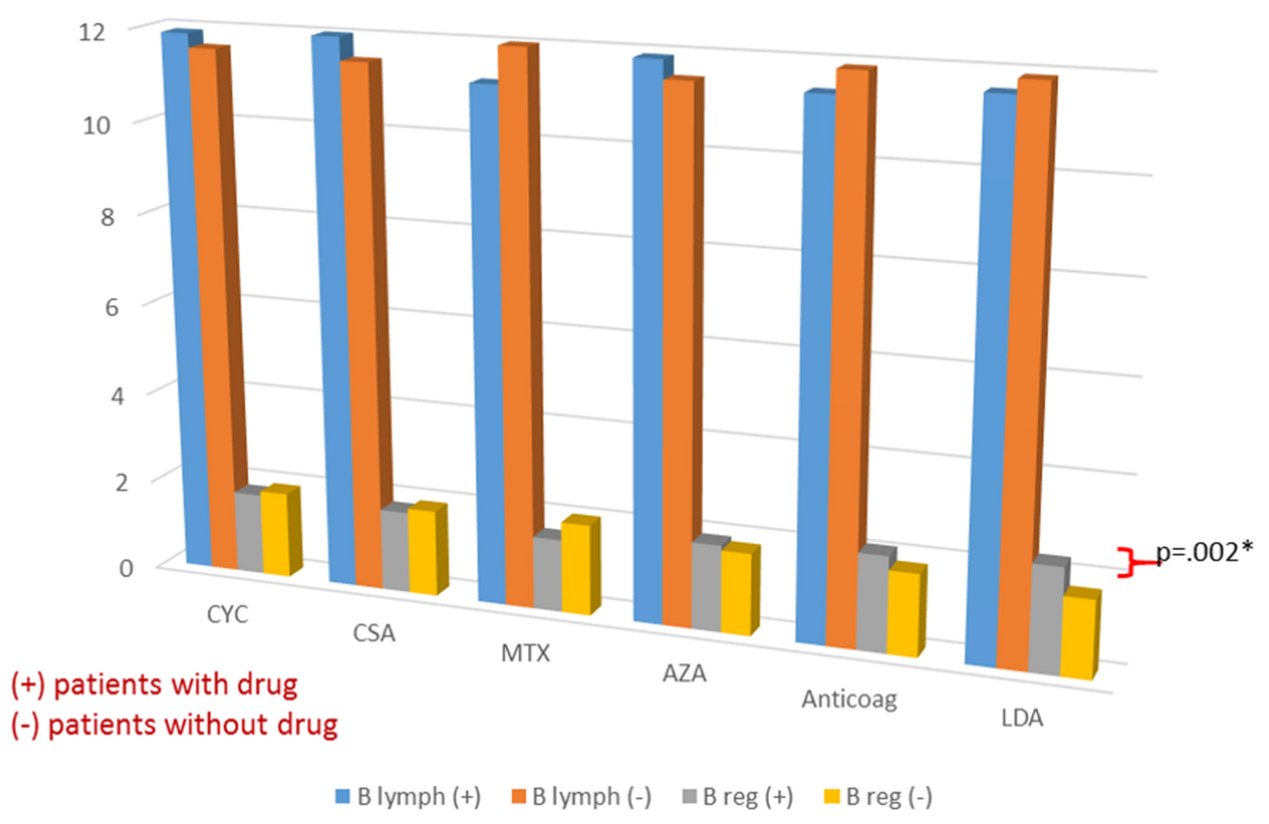

Figure 3 The differences in medians of total B lymphocytes and B regs. Percentages with versus without different drug administration. *Significant difference. Abbreviations: CYC, cyclophosphamide; CSA, cyclosporine-A; MTX, methotrexate; AZA, azathioprine Anticoag; anticoagulant; LDA, low dose aspirin.

inflammation, and normalization of the numbers following anti-TNF therapy. ${ }^{12}$ In this context, the role of B cells in $\mathrm{BD}$ arterial disease was highlighted, previously. ${ }^{28} \mathrm{CD} 20^{+}$ $B$ cells were demonstrated in the vascular wall of a ruptured pulmonary artery aneurysm ${ }^{28}$ suggesting its role in the pathogenesis of arterial disease and mostly explaining the marked drop of these cells in the peripheral blood with this phenotype.

Although there was a lack of association between the clinical activity and ESR, a laboratory marker of inflammation, an increased ESR was linked to a parallel rise in $B$ cells. The value of ESR in determining the activity of different BD manifestations or differentiating the activity of other inflammatory diseases was previously questioned. ${ }^{29,30}$ Besides that, the disease activity scores used for Behcet disease in this article were measuring patients' reported outcomes (PROs). No laboratory work was considered in these scores and the PROs depend on the patients' feelings and perception of disease activity status that could be affected by several personal, psychological and environmental factors regardless of the laboratory landmarks of activity. This might explain the independent association of ESR with increased numbers of $\mathrm{B}$ cells which may be a predictor of a progressive disease rather than an active one.

In the present study, B regs tend to expand in certain clinical presentations, namely, vascular and urogenital diseases. It was noticed that B cells play a role in the resolution of venous thrombosis which was defective after splenectomy or B cell-depletion. ${ }^{31}$ It is possible that they exert a role in the resolution of thrombosis similar to effector T cells. ${ }^{32}$ However, in vitro studies of the regulatory function of $\mathrm{B}$ reg in BD venous and arterial thromboses are warranted to support our findings.

Despite the lack of significant changes in B cell numbers with different therapies, longitudinal studies are required to detect their exact effects along the course of the disease. The observed rise in $\mathrm{B}$ regs in patients receiving LDA is expected to be secondary to the vascular nature of their involvement, which entailed the use of LDA to modify the prothrombotic conditions, rather than an effect of the drug. This was less evident with anticoagulants probably due to the much fewer number of patients on them at the time of recruitment.

The regulatory effect of corticosteroids on $\mathrm{T}$ cell functions has been gaining a lot of attention; ${ }^{33-35}$ nonetheless, their effects on the regulatory function of $\mathrm{B}$ cells have seldom been investigated. The current study strongly suggests a regulatory effect of corticosteroids via upregulating B regs.

One of the limitations of the current work is the observational nature of the study. Small study population is another limitation. Therefore, longitudinal tracking of the dynamic changes in B cells throughout the course of the 
disease in larger patients' number is warranted. It was also unclear, in this study, whether B regs were upregulated with other disease presentations; this is partly due to the scarcity of some involvements, such as different neurological manifestations. In addition, all the patients exhibited oral ulcers and skin lesions, and showed positive pathergy result which hindered to demonstrate the probable upregulation of $\mathrm{B}$ regs in these contexts.

In conclusion, this study supports the role of B cells in $\mathrm{BD}$ pathogenesis and strongly suggests a possible role for $\mathrm{B}$ regs in the resolution of different BD manifestations.

\section{Data Sharing Statement}

Data will be available upon request from the corresponding author.

\section{Acknowledgments}

The authors are thankful to the Assiut University College of medicine grant office for partially funding this work. KM's work is supported by UAE University-start up Grant \# G3347 and UAEU-UPAR-Grant\#G3458.

\section{Disclosure}

The authors report no conflicts of interest for this work.

\section{References}

1. Yilmaz S, Simsek I, Cinar M, et al. Patient-driven assessment of disease activity in Behcet's syndrome: cross-cultural adaptation, reliability and validity of the Turkish version of the Behcet's Syndrome Activity Score. Clin Exp Rheumatol. 2013;31(3 Suppl 77):77-83.

2. Kural-Seyahi E, Fresko I, Seyahi N, et al. The long-term mortality and morbidity of Behcet syndrome: a 2-decade outcome survey of 387 patients followed at a dedicated center. Medicine (Baltimore). 2003;82 (1):60-76. doi:10.1097/00005792-200301000-00006

3. Galeone M, Colucci R, D'Erme AM, Moretti S, Lotti T. Potential Infectious Etiology of Behcet's Disease. Patholog Res Int. 2012;2012:595380. doi:10.1155/2012/595380

4. Kaneko S, Suzuki N, Yamashita N, et al. Characterization of T cells specific for an epitope of human $60-\mathrm{kD}$ heat shock protein (hsp) in patients with Behcet's disease (BD) in Japan. Clin Exp Immunol. 1997;108(2):204-212. doi:10.1046/j.1365-2249.1997.3611265.x

5. Direskeneli H. Innate and adaptive responses to heat shock proteins in Behcet's Disease. Genet Res Int. 2013;2013:249157. doi:10.1155/ 2013/249157

6. de Menthon M, Lavalley MP, Maldini C, Guillevin L. Mahr A. HLAB51/B5 and the risk of Behcet's disease: a systematic review and meta-analysis of case-control genetic association studies. Arthritis Rheum. 2009;61(10):1287-1296. doi:10.1002/art.24642

7. Kirino Y, Bertsias G, Ishigatsubo Y, et al. Genome-wide association analysis identifies new susceptibility loci for Behcet's disease and epistasis between HLA-B*51 and ERAP1. Nat Genet. 2013;45 (2):202-207. doi:10.1038/ng.2520
8. Hasan MS, Bergmeier LA, Petrushkin H, Fortune F. Gamma Delta (gammadelta) $\mathrm{T}$ cells and their involvement in Behcet's Disease. J Immunol Res. 2015;2015:705831. doi:10.1155/2015/705831

9. Puccetti A, Fiore PF, Pelosi A, et al. Gene expression profiling in Behcet's Disease Indicates an autoimmune component in the pathogenesis of the disease and opens new avenues for targeted therapy. J Immunol Res. 2018;2018:4246965. doi:10.1155/2018/4246965

10. Eksioglu-Demiralp E, Kibaroglu A, Direskeneli H, et al. Phenotypic characteristics of B cells in Behcet's disease: increased activity in B cell subsets. $J$ Rheumatol. 1999;26(4):826-832.

11. Suzuki N, Sakane T, Ueda Y, Tsunematsu T. Abnormal B cell function in patients with Behcet's disease. Arthritis Rheum. 1986;29 (2):212-219. doi:10.1002/art.1780290209

12. van der Houwen TB, van Hagen PM, Timmermans WM, et al. Chronic signs of memory B cell activation in patients with Behcet's disease are partially restored by anti-tumour necrosis factor treatment. Rheumatology (Oxford). 2017;56(1):134-144. doi:10.1093/ rheumatology/kew366

13. Davatchi F, Shams H, Rezaipoor M, et al. Rituximab in intractable ocular lesions of Behcet's disease; randomized single-blind control study (pilot study). Int $J$ Rheum Dis. 2010;13(3):246-252. doi:10.1111/j.1756-185X.2010.01546.x

14. Edwards J, Cambridge G, Abrahams V. Do self-perpetuating B lymphocytes drive human autoimmune disease? Immunology. 1999;97(2):188. doi:10.1046/j.1365-2567.1999.00772.x

15. Shimomura Y, Mizoguchi E, Sugimoto K, et al. Regulatory role of B-1 B cells in chronic colitis. Int Immunol. 2008;20(6):729-737. doi:10.1093/intimm/dxn031

16. Abdelwahab FA, Hassanein KM, Hetta HF, Abdelmalek MO, Zahran AM, El-Badawy O. Impact of deranged B cell subsets distribution in the development of HCV-related cirrhosis and HCC in type two diabetes mellitus. Sci Rep. 2020;10(1):1-11. doi:10.1038/ s41598-020-77416-0

17. Hetta HF, Mekky MA, Zahran AM, et al. Regulatory B cells and their cytokine profile in HCV-related hepatocellular carcinoma: association with regulatory T cells and disease progression. Vaccines. 2020;8 (3):380. doi:10.3390/vaccines 8030380

18. Hetta HF, Mwafey IM, Batiha GE-S, et al. Cd19+ cd24hi cd38hi regulatory $\mathrm{b}$ cells and memory $\mathrm{b}$ cells in periodontitis: association with pro-inflammatory and anti-inflammatory cytokines. Vaccines. 2020;8(2):340. doi:10.3390/vaccines 8020340

19. Hassanin KM, Bakr OH, Hetta HF, Mohammed F. B (REG) CELLS IN HEPATITIS C VIRUS AND DIABETES. Bull Pharmaceutical Sci Assiut. 2019;42(1):9-18. doi:10.21608/bfsa.2019.62254

20. Hetta H, Elkady A, Tohamy T, Badary M. Regulatory B cells: key players in hepatocellular carcinoma progression. Gastroenterol Hepatol Open Access. 2016;5(2):00136. doi:10.15406/ghoa.2016.05.00136

21. Yoon JY, Lee Y, Yu SL, et al. Aberrant expression of interleukin-10 and activation-induced cytidine deaminase in B cells from patients with Behcet's disease. Biomed Rep. 2017;7(6):520-526. doi:10.3892/ br.2017.996

22. International Team for the Revision of the International Criteria for Behcet's D. The International Criteria for Behcet's Disease (ICBD): a collaborative study of 27 countries on the sensitivity and specificity of the new criteria. J Eur Acad Dermatol Venereol. 2014;28 (3):338-347. doi:10.1111/jdv.12107

23. Bhakta BB, Brennan P, James TE, Chamberlain MA, Noble BA, Silman AJ. Behcet's disease: evaluation of a new instrument to measure clinical activity. Rheumatology (Oxford). 1999;38 (8):728-733. doi:10.1093/rheumatology/38.8.728

24. International Society for Behçet's Disease. Behçet's Disease Current Activity Form 2006. Leeds: University of Leeds; 2006. 
25. Rhoden EL, Teloken C, Sogari PR, Vargas Souto CA. The use of the simplified International Index of Erectile Function (IIEF-5) as a diagnostic tool to study the prevalence of erectile dysfunction. Int J Impot Res. 2002;14(4):245-250. doi:10.1038/sj.ijir.3900859

26. Garcia-Batista ZE, Guerra-Pena K, Cano-Vindel A, HerreraMartinez SX, Medrano LA. Validity and reliability of the Beck Depression Inventory (BDI-II) in general and hospital population of Dominican Republic. PLoS One. 2018;13(6):e0199750. doi:10.1371/ journal.pone.0199750

27. Ray A, Dittel BN. Mechanisms of regulatory B cell function in autoimmune and inflammatory diseases beyond IL-10. J Clin Med. 2017;6:1. doi:10.3390/jcm6010012

28. Hirohata S, Kikuchi H. Histopathology of the ruptured pulmonary artery aneurysm in a patient with Behcet's disease. Clin Exp Rheumatol. 2009;27(2 Suppl 53):S91-95.

29. Liu S, Ren J, Xia Q, et al. Preliminary case-control study to evaluate diagnostic values of C-reactive protein and erythrocyte sedimentation rate in differentiating active Crohn's disease from intestinal lymphoma, intestinal tuberculosis and Behcet's syndrome. Am J Med Sci. 2013;346(6):467-472. doi:10.1097/MAJ.0b013e3182959a18

30. Muftuoglu AU, Yazici H, Yurdakul S, et al. Behcet's disease. Relation of serum C-reactive protein and erythrocyte sedimentation rates to disease activity. Int $J$ Dermatol. 1986;25(4):235-239. doi:10.1111/j.1365-4362.1986.tb02232.x
31. Frey MK, Winter M-P, Alimohammadi A, et al. Resolution of venous thrombus is depending on B-lymphocytes. Eur Respir J. 2012;40 (Suppl 56):P3909.

32. Luther N, Shahneh F, Brahler M, et al. Innate Effector-Memory T-cell activation regulates post-thrombotic vein wall inflammation and thrombus resolution. Circ Res. 2016;119(12):1286-1295. doi:10.1161/CIRCRESAHA.116.309301

33. Mathian A, Jouenne R, Chader D, et al. Regulatory $\mathrm{T}$ cell responses to high-dose methylprednisolone in active systemic lupus erythematosus. PLoS One. 2015;10(12):e0143689. doi:10.1371/journal.pone.0143689

34. Braitch M, Harikrishnan S, Robins RA, et al. Glucocorticoids increase CD4CD25 cell percentage and Foxp3 expression in patients with multiple sclerosis. Acta Neurol Scand. 2009;119(4):239-245. doi:10.1111/j.1600-0404.2008.01090.x

35. Cepika AM, Marinic I, Morovic-Vergles J, Soldo-Juresa D, Gagro A. Effect of steroids on the frequency of regulatory $\mathrm{T}$ cells and expression of FOXP3 in a patient with systemic lupus erythematosus: a two-year follow-up. Lupus. 2007;16(5):374-377. doi:10.1177/ 0961203307077990
Journal of Inflammation Research

\section{Publish your work in this journal}

The Journal of Inflammation Research is an international, peerreviewed open-access journal that welcomes laboratory and clinical findings on the molecular basis, cell biology and pharmacology of inflammation including original research, reviews, symposium reports, hypothesis formation and commentaries on: acute/chronic inflammation; mediators of inflammation; cellular processes; molecular

\section{Dovepress}

mechanisms; pharmacology and novel anti-inflammatory drugs; clinical conditions involving inflammation. The manuscript management system is completely online and includes a very quick and fair peerreview system. Visit http://www.dovepress.com/testimonials.php to read real quotes from published authors. 